\title{
By-Passing Infected Areas in Wireless Sensor Networks Using BPR
}

\author{
Naimah Yaakob, Ibrahim Khalil, Heshan Kumarage, Mohammed Atiquzzaman, Senior Member, IEEE, and \\ Zahir Tari, Senior Member, IEEE
}

\begin{abstract}
Abnormalities in sensed data streams indicate the spread of malicious attacks, hardware failure and software corruption among the different nodes in a wireless sensor network. These factors of node infection can affect generated and incoming data streams resulting in high chances of inaccurate data, misleading packet translation, wrong decision making and severe communication disruption. This problem is detrimental to real-time applications having stringent quality-of-service (QoS) requirements. The sensed data from other uninfected regions might also get stuck in an infected region should no prior alternative arrangements are made. Although several existing methods (BOUNDHOLE and GAR) can be used to mitigate these issues, their performance is bounded by some limitations, mainly the high risk of falling into routing loops and involvement in unnecessary transmissions. This paper provides a solution to by-pass the infected nodes dynamically using a twin rolling balls technique and also divert the packets that are trapped inside the identified area. The identification of infected nodes is done by adapting a Fuzzy data clustering approach which classifies the nodes based on the fraction of anomalous data that is detected in individual data streams. This information is then used in the proposed by-passed routing (BPR) which rotates two balls in two directions simultaneously: clockwise and counter-clockwise. The first node that hits any ball in any direction and is uninfected, is selected as the next hop. We are also concerned with the incoming packets or the packets-on-the-fly that may be affected when this problem occurs. Besides solving both of the problems in the existing methods, the proposed BPR technique has greatly improved the studied QoS parameters as shown by almost 40 percent increase in the overall performance.
\end{abstract}

Index Terms-Wireless sensor networks, routing protocols, fault-tolerance, anomalies detection, performance evaluation

\section{INTRODUCTION}

W IRELESS sensor networks (WSN) have been the cutting-edge technology in various remote event monitoring applications, especially in hazardous areas and hostile environments, for more than a decade [9]. The detection of certain events is made viable through data sensing and forwarding from sensor nodes to the socalled sink node for further processing [18]. As such, the occurrence of any unexpected circumstance normally involves communication of outstanding data to the sink node. However, energy constraints and other resource limitations [27] restrict direct communications between sensors and the sink node. Therefore, communications in WSN are affected by the proper functionality and state of various intermediate nodes which in turn forward the received data to another node until they reach their destination. This saves colossal amounts of energy in each

- N. Yaakob is with the School of Computer Science and IT, RMIT University, Swanston St, Melbourne, VIC 3000, Australia, and the School of Computer and Communication Engineering, Universiti Malaysia Perlis (UniMAP), Malaysia.E-mail: naimahyaakob@unimap.edu.my.

- I. Khalil, H. Kumarage, and T. Zari are with the School of Computer Science and IT, RMIT University, Swanston St, Melbourne, VIC 3000, Australia.E-mail: \{ibrahim.khalil, zahir.tari\}@rmit.edu.au, heshan_kumarage@student.rmit.edu.au.

- M. Atiquzzaman is with the School of Computer Science, The University of Oklahoma, Norman, OK 73019. E-mail: atiq@ou.edu.

Manuscript received 30 Jan. 2014; revised 15 July 2014; accepted 16 July 2014. Date of publication 4 Aug. 2014; date of current version 13 May 2015.

Recommended for acceptance by I. Stojmenovic.

For information on obtaining reprints of this article, please send e-mail to: reprints@ieee.org, and reference the Digital Object Identifier below.

Digital Object Identifier no. 10.1109/TC.2014.2345400 node and prolongs their battery lifetime while maintaining persistent connectivity.

\subsection{Problem Statement}

Due to their limited capabilities, sensor nodes are susceptible to various sources of failure. These include malware attacks, hardware failures and software corruption which can reduce nodes' functionality and badly affect most WSN operations [24]. These threats could lead to critical drawbacks such as partial or complete node failure that causes destructive effects on the underlying monitoring applications. Nodes experiencing such failures or malfunction can be classified as infected and will normally fail to perform normal sensing and communication tasks. Hence, they are unable to observe the timely delivery service which is crucial to maintaining high quality-of-service (QoS) in WSN.

Among the most threatening effects of the afore-mentioned problem is the high tendency for the infected nodes to produce faulty data. An example of infected nodes and regions on a WSN is shown in Fig. 1. Data generated by this kind of nodes may contain anomalies that do not resemble the actual data and differ from the expected readings [2]. For example, a set of a sensed temperature readings can contain several fluctuations that are totally different from the normal temperatures observed as the majority.

When a node malfunctions, the entire sensing processes may be disturbed, causing serious intermittent connection over the entire network. During such a situation, some packets may not be forwarded to their destinations; may become lost in transmission or get stuck in an infected area. 


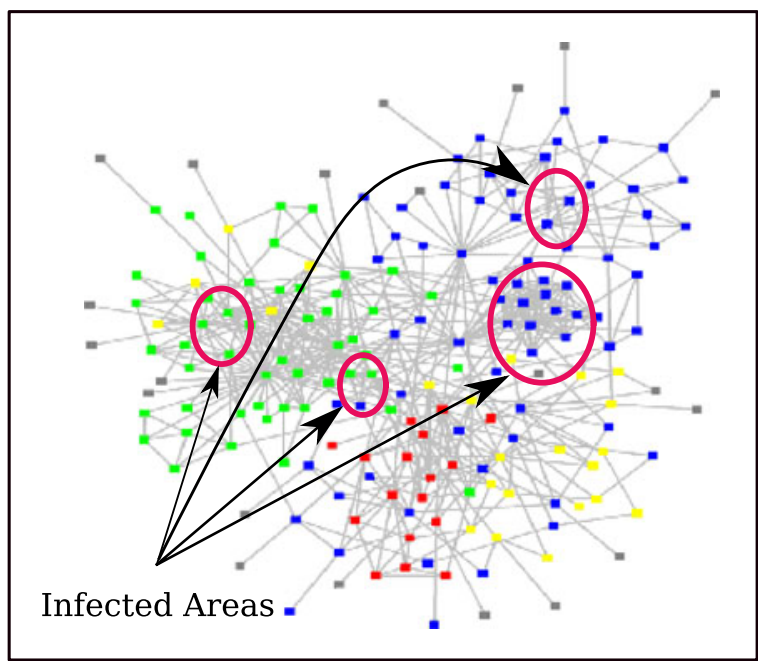

Fig. 1. The scenario when some nodes are identified as infected due to malware attack, hardware failure or software corruption, which can cause substantial performance degradation.

Such problems will introduce a rise in the packet loss rate and a higher consumption of energy which are the major concerns in WSN that suffer from resource limitations. Some of these packets may carry significant information about the occurrence of any emergency situation. The loss of this kind of packets could result in severe consequences that may affect the whole industry or nation utilizing the network. Packets containing anomalous data can also result in false analyses and in-correct decision making at the end systems. Hence there is an imperative need to timely detect infected nodes and avoid them in subsequent communication and transmission procedures. This requires fast alternative routes to be reconstructed in order to detour any incoming packets to their destinations. This could be achieved by effective avoidance of infected areas.

\subsection{Objectives}

This research is driven by a concern to address the following objectives:

- To avoid packets from being stuck in any identified infected region and find a solution to get trapped packets out from already infected areas. This aims to minimize the number of packets trapping and information loss in different network areas and mitigate the negative impact on the underlying decision making systems which could be massive.

- To design a method that can by-pass infected areas and redirect the incoming traffic to the unaffected regions.

\subsection{Contributions}

The proposed solution in this paper is twofold and can be summarized as follows. First, the specific infected nodes are identified using a fuzzy data clustering approach that partitions the data into groups in order to identify anomalous data comprising both individual outliers and clustered groups. A node that contains a significant fraction of anomalous data, is said to be infected. Second, this kind of node is to be avoided in subsequent routing procedures that comprises the main contributions in this paper and can be noted as follows:

- An innovative way of reducing the increase in packet loss rate is proposed by avoiding or by-passing the identified infected regions and redirecting the packets using the uninfected nodes. This method is called by-passed routing (BPR).

- Uniquely, in order to get the stuck packets out of infected areas, a unique twin rolling balls (RBs) technique is proposed in which two balls are rotated clockwise and counter-clockwise simultaneously. Different from existing rolling ball technique [25], the next hop is selected when a node is touched by one of the balls, provided that it is uninfected.

- A mechanism to route packets-on-the-fly away from the identified regions is proposed since they may have a high tendency to get trapped and lost. These packets will be detoured using different nodes so they can safely reach their destinations. It is crucial to the detection and classification of the infected data to recognize which nodes they belong to in order to divert the traffic away from the identified regions.

Performance evaluation and analysis using network simulator 2 (NS-2) show that the proposed solution is able to facilitate fewer hops traversed, a higher packet delivery ratio (PDR) and excellent routing efficiency. The proposed BPR method is also able to minimize the average end-toend delay and reduce a considerable amount of communication overhead. Overall, our technique shows desirable performance and exhibits substantial improvement compared to existing methods.

The rest of the paper is organized as follows. Section 2 presents several related work found in literature, while Section 3 formulates the problem. The paper continues with the proposed solutions in Section 4 which consists of two parts: Fuzzy data clustering based detection for infected nodes and by-passed routing. The proof of the concept is given in Section 5, followed by the experimental evaluations in Section 6. Finally, Section 7 concludes the paper.

\section{Related Work}

There are few inherent issues that concern WSN research communities including fault-resilience [17], network lifetime [1], sensor localization [26], sink mobility [16], security [14], [22] and also routing [10], [25]. Among all, routing issues have been receiving the most significant interests.

Most of the routing protocols developed for sensor networks employ greedy forwarding (GF) algorithm [5] which forwards a packet to a destination node via 1-hop neighbour [4], [13]. The neighbour that receives the packet will repeat the process until the packet reaches its intended destination. This technique is proven to be efficient in reducing energy consumption since it does not incur additional routing overhead. However, it suffers from the local minima phenomena or 'holes' problem which has attracted much attention from the research communities in the sensor network domain, resulting in various methods as found in [3], [5], [7], [8], [15], [28].

1. Packets that have already been transmitted without knowing that the nodes used in their route are infected. 
Local minima generally refers to the the classic situation where packets cannot be forwarded to the next hop since there exists no other node that has shorter distance to destination than itself. Some of these methods employ a graphbased technique, which require the entire network graph to be stored, thus leading to poor scalability. On the other hand, non-graph-based techniques will result in long routing paths and high energy expenditure, which are unsuitable for WSN. Here, holes can also be inferred as the hot spots' caused by traffic congestion, or the phenomenon where sensor nodes are destroyed due to natural disasters such as forest fire or nodes failure. The presence of holes introduces several difficulties and may cause major changes in the network topology.

Another issue in the traditional GF is congestion [6], [23]. Since sensors in WSN are densely deployed, some nodes may end up transmitting to the same hop, thus causing traffic overflow. This scenario will severely degrade the whole network performance. One way to tackle this issue is by using multiple paths technique [20] and load balancing [11]. The latter approach deploys geographic position information and a network congestion metric to balance the traffic so that congestion is significantly reduced.

Nevertheless, our method is focusing on a routing technique to divert traffic away from any holes or infected regions. Basically, the idea of by-passing the holes can be found in [10]. This approach introduces BOUNDHOLE algorithm to discover holes and establish adaptive routes to by-pass the identified nodes. This algorithm separates the boundary of the holes and routes the packets based on the original GF. The communication between boundary nodes is done using sweeping lines, which requires nodes to remember the shape of the previous holes, thus needs extra memory. Moreover, holes can always be dynamic in nature and the use of the previous holes' shapes may just be wasting the limited memory and energy of sensor nodes. Another major issue of BOUNDHOLE is the false boundary detection problem that presents a high risk of falling into a loop. This leads to longer routing which may quickly deplete the energy of the nodes and severely degrade the performance.

Greedy anti-void routing (GAR) [25] has been designed to tackle the issue of false boundary detection in the BOUNDHOLE approach. It employs a rolling ball method which is attached or hinged at the node having the local minima problem and rotate counter-clockwise with $R / 2$ radius. The first node that intersects with the rolling ball and is closer to the destination node will be selected as the next hop. The ball will then continue the rotation until the next node is hit and the process continues until the packet safely arrives at the destination node. Though this method exhibits better performance than BOUNDHOLE, it visits unnecessary nodes, resulting in higher energy consumption. Our proposed technique is basically similar to the rolling ball technique used in GAR. However, it eliminates visits to unnecessary nodes, resulting in a shorter routing path and a more efficient routing protocol.

\section{Problem Formulation}

This section explains the problem formulation of this paper. All the notations used can be referred in Table 1. Consider a
TABLE 1

List of Notations

\begin{tabular}{lll}
\hline Number & Notation & Translation \\
\hline 1 & $N$ & Set of Sensor Nodes \\
2 & $N_{s} \& N_{D}$ & Source and Destination Node \\
3 & $N_{j} \& N_{i}$ & Next and Current Node \\
4 & $N_{\text {Local }}$ & Local Minima Node \\
5 & $\delta T$ & Time Window \\
6 & $X$ & Set of Observations \\
7 & $J_{m}$ & Objective Function \\
8 & $C_{j} \in R$ & Set of Cluster Centroids where \\
& & $R$ is a real number \\
9 & $W_{i j}$ & Membership degree of \\
& & point $X_{i}$ in jth cluster \\
10 & $T=\left[W_{i j}\right]$ & Fuzzy partition of $X$ \\
11 & $P_{N i}\left(X_{N_{i}}, Y_{N_{i}}\right)$ & Statistical Threshold \\
12 & $R$ & Position of Node $N_{i}$ \\
13 & $R / 2$ & Radius of a circle \\
14 & $d_{c}$ & Radius of a Rolling Ball \\
15 & $d_{c c}$ & Clockwise direction \\
16 & $S_{i}$ & Counter-Clockwise direction \\
17 & $d\left(N_{i}, N_{D}\right)$ & Centre Point of RB \\
18 & & Distance from node $N_{i}$ \\
& $D$ & to destination node $N_{D}$ \\
19 & $T_{N}$ & Node's transmission range \\
20 & $I D_{N}$ & Neighbouring Table \\
21 & & ID of each node \\
\hline & &
\end{tabular}

set of nodes $N=\left\{N_{i} \mid \forall i\right\}$ within a two-dimensional (2D) euclidean plane. The location of the destination node $N_{D}$ is known in advance by the source node $N_{S}$. The locations of other nodes in the set are known using the periodic beacon updates. These positions can be presented by $P=\left\{P_{N} \mid\right.$ $\left.P_{N}\left(x_{N i}, y_{N i}\right), \forall i\right\}$. We assume that all the sensor nodes are homogeneous. A set of $N$ nodes' transmission ranges can be denoted by $\bar{D}=\left\{\bar{D}\left(P_{N i}, R\right) \mid \forall i\right\}$ where $\bar{D}\left(P_{N i}, R\right)=$ $\left\{x \mid\left\|x-P_{N i}\right\| \leq R, \forall x \in R^{2}\right\}$. In this case, the transmission range for each of $N_{i}$ is given by $R$ and the centre of the radius is denoted by $S_{N}$. Since the packet is conveyed to destination using the 1-hop information as in GF [5], the neighbouring table is given by $T_{N_{i}}=\left[I D_{N_{k}}, P_{N_{k}}\right] \mid P_{N_{k}} \in$ $\bar{D}\left(P_{N_{i}}, R\right), \forall k \neq i$ where $I D_{N_{k}}$ represents the identification number for node $N_{k}$. To initiate a transmission, a source node $\left(N_{S}\right)$, knowing the location of the destination node $\left(N_{D}\right)$, will choose the next hop from its routing table $T_{N_{s}}$ that has the distance closer to the destination node than itself. The same procedures repeated until all the packets have been received by the destination node.

\subsection{Local Minima Problem}

Local Minima or void is a problem derived from the original GF algorithm. It can be defined as follows:

Problem 1 (Local minima) [19]. The local minima problem occurs when there exists a node $N_{v}$ in the network, that has no closest 1-hop neighbour to the destination than itself. In such a scenario, the node cannot forward the packets to their destinations, hence creating the local minima problem. This can be presented as follows:

$$
\left\{P_{N_{k}} \mid d\left(P_{N_{k}}, P_{N_{D}}\right)>d\left(P_{N_{v}}, P_{N_{D}}\right), \forall P_{N_{k}} \in T_{N_{v}}\right\}=\phi .
$$




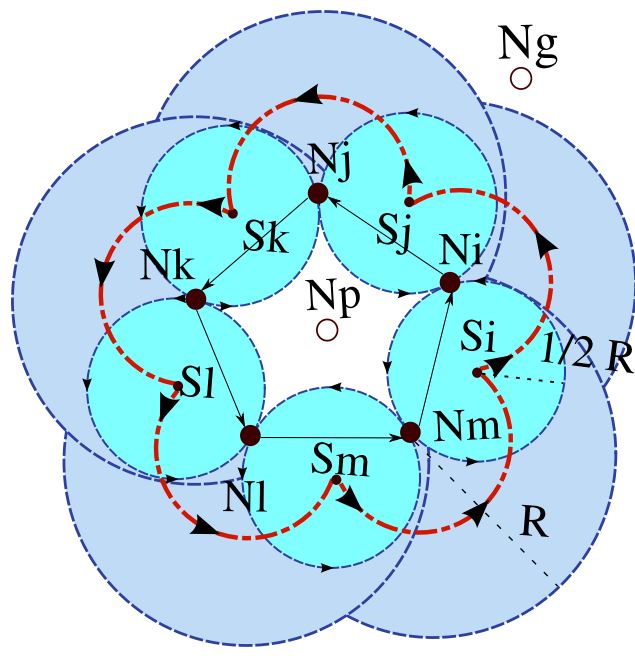

Fig. 2. The rolling ball operation.

Where $T_{N_{v}}$ is the neighbouring table containing the closest 1hop neighbours of $N_{v}$. In this case, $N_{k}$ could not be chosen to be the next forwarding hop since it has larger distance to destination compared with $N_{v}$.

\subsection{The Rolling Ball Limitations}

The RB can be illustrated in Fig. 2. While RB is proven to be successful in avoiding the identified infected regions, it tends to visit unnecessary nodes and results in longer routing delays. The definition of $\mathrm{RB}$ and its problem can be given as follows:

Definition 2 (Rolling Ball) [25]. In a given set of sensor nodes $N_{i} \in N_{r}$, we say a circle is a Rolling Ball if there exists a circle $\left(R B_{N_{i}}\left(S_{i}, R / 2\right)\right)$ that is hinged or attached at a centre point $S_{i} \in R^{2}$ with a radius of $(R / 2)$. No other node such as $N_{k} \in N$ should be located inside the rolling ball as $\left\{R B_{\bar{N}}\left(S_{i}, R / 2\right) \cap N\right\}=0$ where $R B_{\bar{N}}\left(S_{i}, R / 2\right)$ denotes the open area within the rolling ball.

Problem 2 (False Boundary Detection). As the ball $\left(R B_{\bar{N}}\left(S_{i}\right.\right.$, $R / 2)$ ) is hinged at the centre of the node $N_{i}$, it will rotate in a clockwise or counter-clockwise until the next node $N_{j}$ touches the rolling ball. The same procedures apply to $N_{j}$ until the ball enters a termination phase. However, there will be communication intersection with another nodes as shown in Fig. 3. This will result in longer routing path due to visiting the unnecessary nodes.

\section{The Proposed Solution}

The proposed by-passed routing technique comprises two main parts, namely Infected area detection and by-passed routing. The first part detects the occurrence of infected nodes adapting a Fuzzy Data Clustering approach to identify anomalies based on the received data signals. The fuzzy clustering method is chosen as it provides an unsupervised and modular method for evaluating anomalous data over the different sensor nodes. A data-centric point of view as with fuzzy clustering is suitable when evaluating whether a node is infected or not, whether it is through a hardware malfunction, malware attack or software corruption.

The information about infected nodes is then directly used for traffic diversion in the proposed BPR technique.

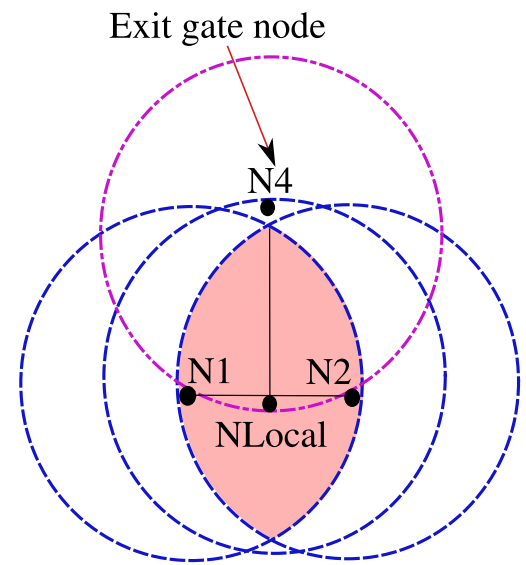

Fig. 3. The communication intersection problem which defines the exit gate node.

The novelty of the BPR approach relies on the introduction of the simultaneous twin rolling balls technique that detects the next 1-hop neighbour faster than the existing GAR approach. Using this approach, the first node that hits any ball in any direction and is uninfected is assigned as the next hop. A further different way of getting the stuck packets out of infected regions is another unique contribution of BPR.

This section begins with the details of the fuzzy data clustering approach for infected area detection before proceeding to the proposed BPR technique, which is the heart of the paper. The overall picture of the proposed BPR technique is shown in Fig. 4.

\subsection{Fuzzy Data Clustering}

Here, we define a particular sensor node as an infected node and a set of such nodes which are adjacent to each other in terms of communication space as an infected area as per the following definitions.

Definition 3 (Infected Node). Given a set of sensor nodes $N=\left[N_{1}, N_{2}, \ldots, N_{n}\right]$ on a WSN, a particular node $N_{i}$ is considered as infected if it satisfies the following condition.

- Contains some outlying fluctuation points which can be classified as anomalous with it's fraction over normal measurements is $\geq 10$ percent of its aggregated readings over a considered time window $\delta T$.

Definition 4 (Infected Area). Given a subset ( $n$ ) of WSN sensor nodes $(n \in N)$, which are over a particular spatial area $A$, that area is considered as an infected area if and only if;

- All the sensor nodes in $n$ satisfy the criteria for Definition 3.

- Each node is within one hop communication distance of at least one other node in $n$.

The actual detection of anomalies in sensor measurements is performed by adapting the Fuzzy C-means algorithm for data clustering and classification based on the work in [12]. Therefore, soft data partitioning (clustering) is performed in an unsupervised manner at each of the sensor nodes in the network for the same defined time window of $\delta T$. For a set of observations $X=\left[X_{1}, X_{2}, \ldots, X_{n}\right]$, where each data point $X_{i}$ is a $d$-dimensional measurement 


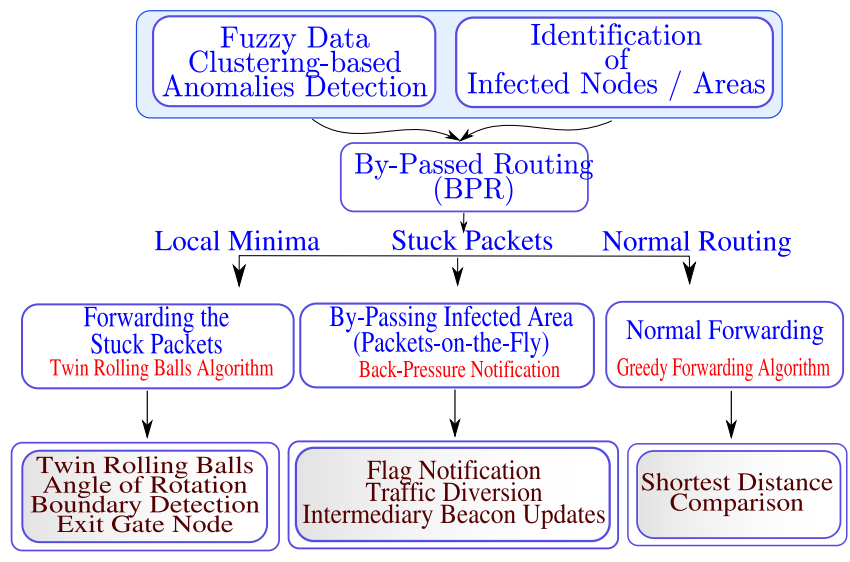

Fig. 4. The proposed architecture view for by-passed routing technique.

collected within the considered time window, the following objective function $\left(J_{m}(U, C ; X)\right)$ is minimized as the weighted sum of squared errors according to the Fuzzy Cmeans algorithm;

$$
J_{m}(U, C ; X)=\sum_{j=1}^{k} \sum_{i=1}^{n} w_{i j}^{m}\left\|x_{i}-c_{j}\right\|_{A}^{2}, 1<m<\infty .
$$

Here, $C=\left(c_{1}, c_{2}, \ldots, c_{k}\right)$ is the set of cluster centroids with each $c_{j} \in R$. The membership degree for data point $X_{i}$ in the $j$ th cluster is denoted by $w_{i j}$. The euclidean distance is chosen as the similarity measure between a particular data point and the cluster centroid as it provides an effective similarity score with low computational cost. This is defined in the inner product with norm matrix $A$. Therefore a fuzzy partition of $X$ is derived by the representing matrix $U=\left[w_{i j}\right]$ consisting of all membership values.

The clustering operation is performed with a userdefined number of expected clusters. As the work in [12] elaborates, there is little effect in the number of clusters with regard to identifying the anomalies when used over an expected middle range of (6-12). This is a valid argument, especially given the small temporal window that the data is aggregated over. Therefore, this technique sets the number of expected clusters as eight (8) with regards to avoiding large computational overheads as well as being representative enough for covering the distribution of data over a small temporal window. Thus, each node produces a set of fuzzy values $U=\left[w_{i j}\right]$ indicating the membership degree of its data points to each cluster from the determined set of eight centroids.

$$
T=1 / n \sum_{i=1}^{n} X_{i} \pm \sqrt{1 / n \sum_{i=1}^{n}\left[X_{i}-\left(1 / n \sum_{i=1}^{n} X_{i}\right)^{2}\right]} .
$$

As the next step, the soft clustering is de-fuzzified by introducing adaptively derived statistical thresholds at each node. This threshold $(T)$ is given in the above equation with $X$ representing any data set to which it is applied. Therefore, one standard deviation away from the mean with regard to the set of maximum membership values from the obtained fuzzy partition $\left[\max U\left(w_{i j}\right)\right]$ is used to initially identify the anomalies. Data points that cannot be satisfactorily attributed to a defined cluster centroid using the Fuzzy C-means algorithm is isolated in this step.

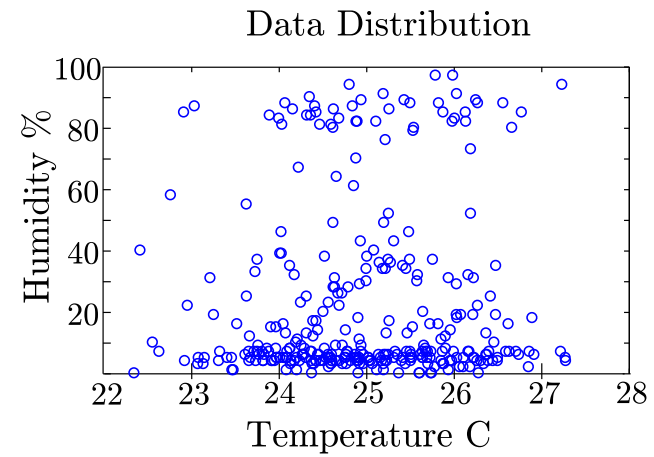

(a)

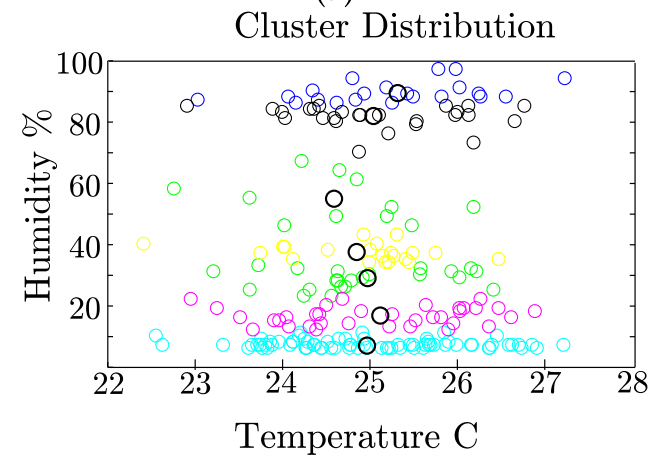

(b)

Fig. 5. (a) Data distribution of normal temperature/humidity readings (no anomalies). (b) The resulting groups of clusters using the proposed fuzzy clustering approach for anomaly detection. Each colour represents membership in a different cluster.

While this gives the anomalous data points that are characterized by their non-membership in the defined clustering, we are also interested in obtaining the anomalous data that are composed on to the identified clusters themselves. This is performed by introducing the same non-parametric threshold given above to a different set of values. Specifically, we evaluate the abnormality of the formed clusters through thresholding the mean inter-cluster distance among the eight defined cluster centroids at each sensor node. Therefore, if the mean inter-cluster distance of a particular centroid falls above the threshold for the set of mean inter-cluster distances, that cluster itself is considered as anomalous. The combination of the two techniques in identifying anomalies by evaluating data points for cluster membership and distance comparison for centroids provides the final anomaly count at each node. Sample data distributions for two instances containing normal and anomalous readings (in this case for temperature and humidity) and the results of the proposed clustering and anomaly detection are shown in Figs. 5 and 6 respectively.

Once this indicates that a particular node comprises of 10 percent or more of anomalous data within a considered period, that node is marked as infected. We use 10 percent as the cut-off due to the fact that it is the lowest value possible to have while still allowing for some flexibility with regard to occasional fluctuations in the data that can be detected as anomalies. This information on detected infection is then shared among the immediate 1-hop neighbours of each node which will then be used in the proposed bypassed routing module in the next section. 
Data Distribution

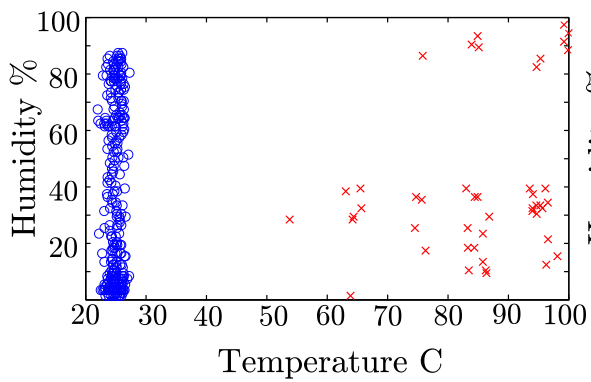

(a)

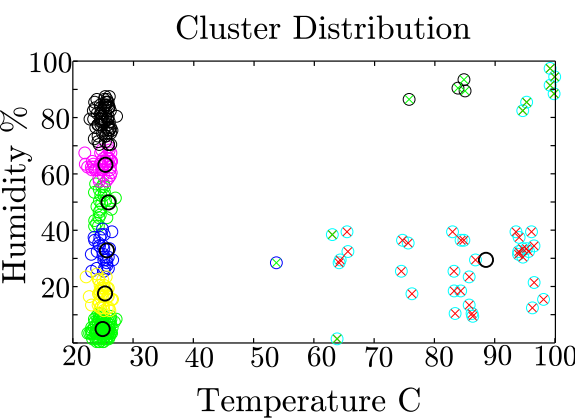

(b)

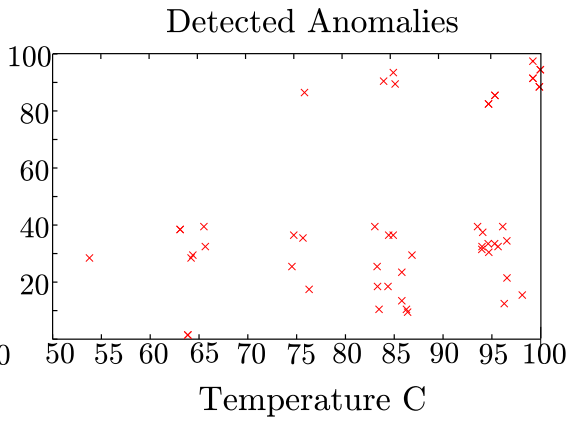

(c)

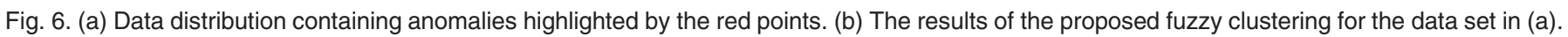
(c) Identified anomalies using the proposed method for the data set in (a).

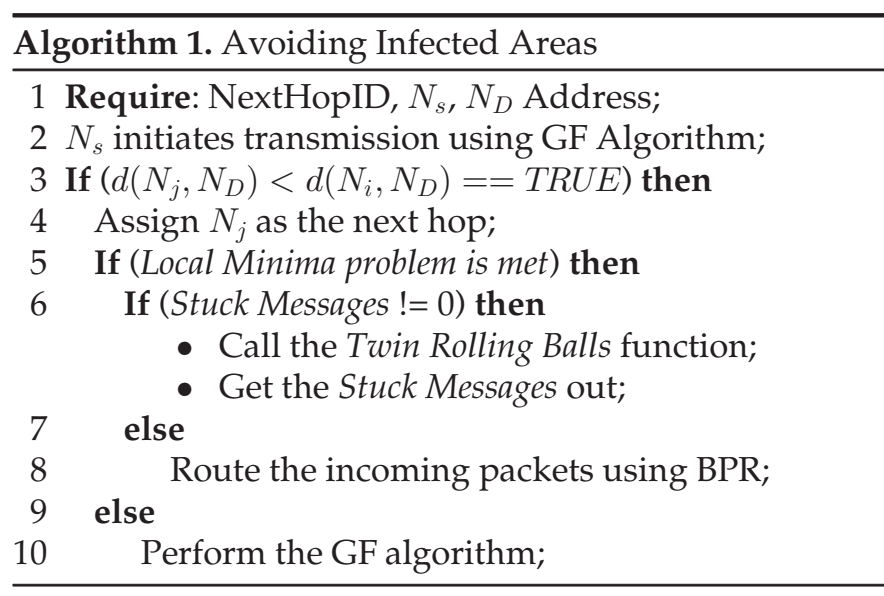

\subsection{By-Passed Routing}

The aim of this technique is twofold. First, to get the stuck or trapped packets out of the corrupted regions in a timely manner by observing real-time applications maximum delays of $150 \mathrm{~ms}$. Second, we are also concerned about the incoming traffic that will have to be detoured in order to avoid them from being sent to the infected region. Once the information about the infected nodes is obtained, the information is used to by-pass the area and redirect incoming traffic to unaffected nodes. Having said that, this section comprises of three different parts: Getting the Stuck Packets Out, By-passing the infected areas, and Normal Routing.

\subsubsection{Getting the Stuck Packets Out}

When nodes are infected, some packets are trapped inside the region and cannot be forwarded to the next hop simply because there is no available node to do so. These packets may have a high possibility of being dropped if no alternative arrangements are made to get them out of the infected region.

This section explains the detailed implementation of the proposed technique which consists of three parts: the proposed Twin Rolling Balls, Forwarding the Stuck Packets, and the Derivation of Exit Gate Node. The whole process of avoiding the infected region is presented in Algorithm 1.

The twin rolling balls. Once the infected packets and the nodes that they are residing have been identified, we need to define the boundary nodes to route the packets away from the infected areas. The detection of the boundary nodes in the proposed method is inspired by the rolling ball [25] technique as described in Section 3.2. However, unlike the previous method [25], the angle of rotations are in both directions; clockwise $\left(d_{c}\right)$ and counter-clockwise $\left(d_{c c}\right)$ as shown in Fig. 7. Rotating in just one direction may take a longer time if the node happened to be located far away from the ball.

We counter this problem using two different balls that are attached to the same point $\left(N_{\text {Local }}\right)$ and rotate the balls in different directions. The proposed algorithm does not just consider the intersection between the rolling ball $\left(R B i_{N i}\left(S_{i}, R / 2\right)\right)$ and a node as the next corresponding boundary node, but also the absence of anomalies in the potential forwarding node. Besides avoiding a close-loop formation and false-boundary detection, this approach automatically avoids packets from being forwarded to the wrong nodes as in BOUNDHOLE. Furthermore, the proposed twin rolling balls ensures faster detection of the next hop since the node can be hit by any of the balls in any directions. For example, if a node is located closer to the ball in clockwise direction, rotating the ball in counter-clockwise may result in longer delay. We define the following properties.

Definition 5 (The Twin Rolling Balls). The Twin Rolling Balls is defined as two identical balls $R B 1_{N_{i}}\left(S_{i}, R / 2\right)$ and $R B 2_{N_{i}}\left(S_{i}, R / 2\right)$ each with radius $R / 2$ hinged at $N_{\text {Local }}$ and rotate in two directions simultaneously until the first node is hit. The twin rolling balls operation can be found in Algorithm 2.

Definition 6 (Exit Gate Node). We exemplify a node $N_{x}$ as an exit gate node with the angle of $\angle N_{1} N_{2} N_{\text {Local }}$ in such a way that $N_{x}$ is in the transmission range of $N_{\text {Local }}$ and out of transmission range $N_{1}$ and $N_{2}$.

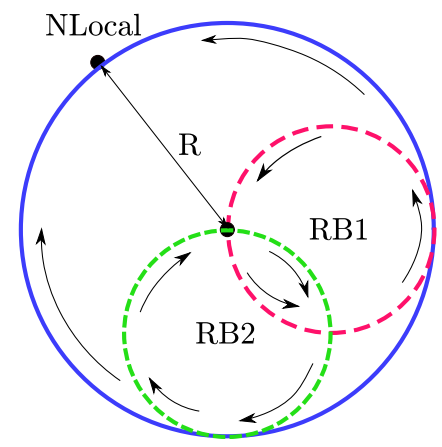

Fig. 7. The twin rolling balls operation. 


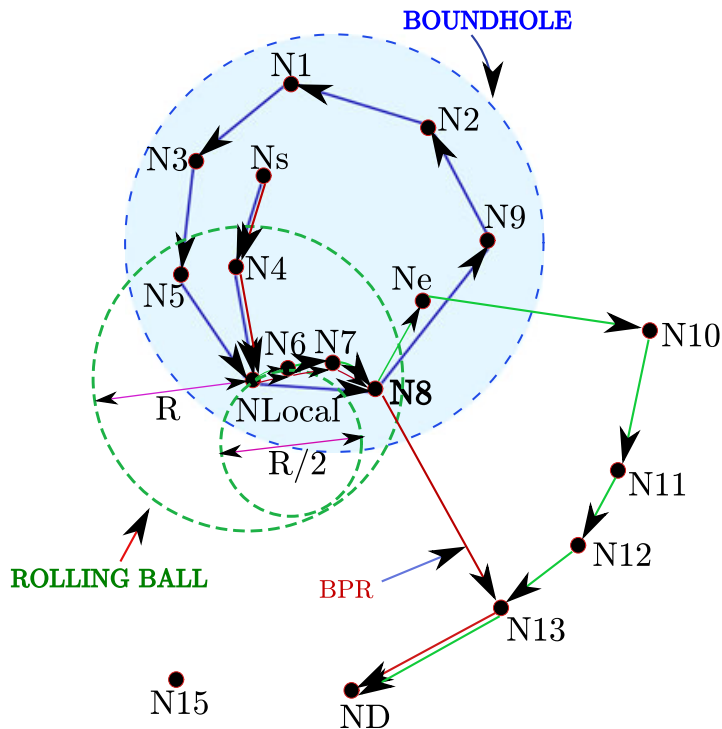

Fig. 8. Example of the constructing path using BOUNDHOLE and Rolling Ball Algorithm.

\begin{tabular}{l} 
Algorithm 2. The Twin Rolling Balls \\
\hline 1 Require: $N_{\text {Local }}$ location and radius $R ;$ \\
2 If $\left(d\left(N_{\text {Local }}, N_{D}\right)<\left(N_{i}, N_{D}\right)==T R U E\right)$ then \\
- Attach 2 rolling balls $R B 1_{N_{i}}\left(S_{i}, R / 2\right)$ and \\
$R B 2_{N_{i}}\left(S_{i}, R / 2\right)$ at the center of $N_{\text {Local }}$. \\
• Rotate the balls in both directions \\
If (Any ball hits any node) \&\& (Nodes != \\
Anomalies) then \\
- Assign that node as the new hop $N_{i} ;$ \\
- $N_{\text {Local }}$ will pass the packets to $N_{i} ;$ \\
- Repeat all the steps until $N_{\text {exit }}$ is found \\
- ExitGateNode ()$;$ \\
3 else \\
Route the packets using normal GF algorithm;
\end{tabular}

Forwarding the stuck packets. In getting the stuck packets out from any infected regions, we based our analysis on BOUNDHOLE [21] and GAR [25]. Having said that, the path selection using our approach results in shorter path diversion. This is because we avoid visiting the unnecessary nodes that will lead to an undesirable longer routing path. Unlike the existing rolling ball technique, once the local minima occurs, there will be two balls attached at the local minima node that rotate in two directions simultaneously; clockwise and counter-clockwise. This method will then compare the distance of the first node that hits the rolling ball from both directions. Referring to Fig. 8, the first node that hits the ball in clockwise is $N 5$, while for counter-clockwise is $N 6$.

Our method suggests that the first node that hits the ball in either direction and is not infected, will be chosen as the next hop ( $N 6$ using counter-clockwise rotation). This node also determines the direction for the rest of the rotation. The ball continues moving counter-clockwise and the same process continues until all the nodes within the communication range $(R)$ of $N_{\text {Local }}$ are used. This includes the nodes that originated the packet (e.g. source and intermediate nodes). By avoiding unnecessary transmissions, this method can result in shorter paths, save much energy and so prolong network lifetime.

The exit gate node $\left(N_{\text {exit }}\right)$. The process of finding an exit gate node can be found in Algorithm 3. As shown in Fig. 8, the last node that hits the ball is $N 8$. If we select the previously used nodes, the close-loop routing will occur as in BOUNDHOLE method. Since the last node that hits the ball is $N 8$ and it contains no anomalies, we regard it as an Exit Gate node $\left(N_{\text {exit }}\right)$, and this is contrast with GAR method which chooses $N_{e}$. As the ball hinges at $N_{e}$, it will rotate and hit $N_{10}$ and this process continues until the packet reaches its destination node $N_{D}$. Not surprisingly, this method introduces unnecessary visits to other nodes $\left(N_{e}, N_{10}, N_{11}, N_{12}\right)$ whilst there is a shorter route to destination.

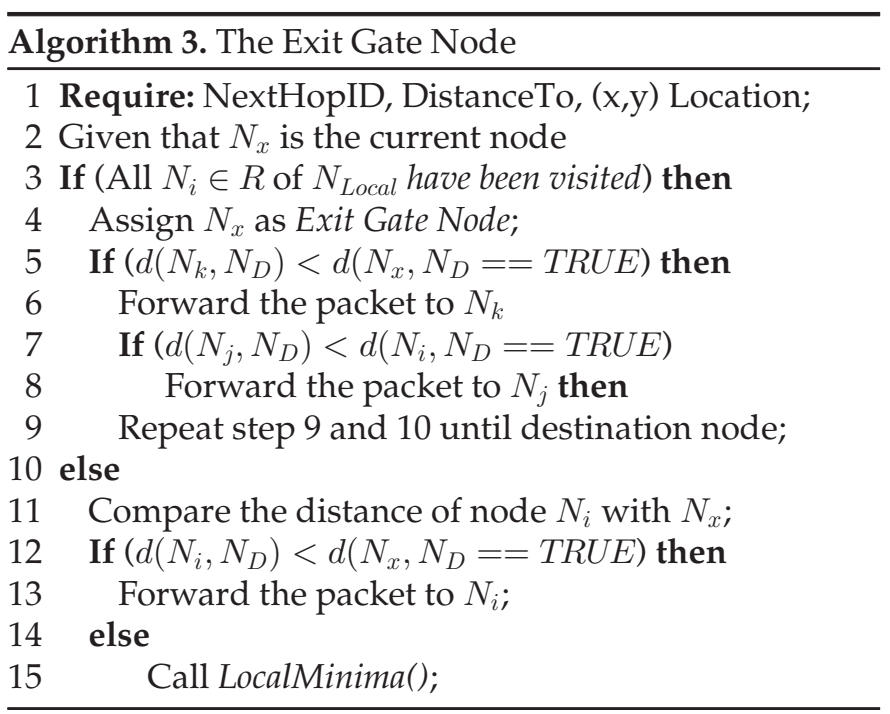

In contrast, the selection of $N 8$ in our method is based on the transmission range covered by $N_{\text {Local }}$. For this reason, we exclude $N_{e}$ as the potential exit node and therefore avoid visiting the longer route. At this point, we proceed with the normal forwarding method (GF). Since none of the neighbouring nodes are inside the communication range of $N_{\text {Local }}$, the next hop is obtained by comparing the shortest distance between $N e, N 9$ and $N 13$ to the destination $N_{D}$. However, since $\mathrm{Ne}$ and $\mathrm{N} 9$ are located further away from $N_{D}, N 13$ will be chosen to forward the packets to the destination node based on the information received from $N 8$. The total number of visited hops using this technique is 7 in contrast with 11 by the GAR. Thus, we save considerable time and resources by avoiding the transmission through unnecessary nodes.

\subsubsection{By-Passing Infected Areas}

Considering the aforesaid significance of providing timely delivery of real-time packets, this method acknowledges the need to protect both generated and packets 'on-the-fly' from being routed to infected nodes. Therefore, we provide an alternative route to detour the affected packets.

The initial phase of the proposed BPR technique is based on the GF algorithm. Neighbours' location and distance to other neighbours are obtained through frequent beacon updates and kept in each node's routing table. There are three processes in this method. First is flag notification of 
the infected nodes. This is followed by traffic diversion and finally the beacon updates.

Flag notification of the infected nodes. Through the Fuzzy data clustering technique, each node is aware of their infection status. Once infected, the corresponding node will quickly notify the source node so that it will no longer receive any incoming packets. This is done via a back-pressure notification by triggering an infection flag in the notification packet and sending it to the source node. Here, the flag is set to 1 if there is any infection, and stays 0 in normal mode.

The back-pressure is a message sent backwards to notify the senders of any events. This will also involve intermediate nodes that reside within the same route with the affected nodes. Upon receiving this notification, source node will stop sending through the infected node. The notification is done using back-pressure method instead of broadcasting since the latter will send notifications to all the nodes in the network. This will result in unnecessary transmissions and obviously waste valuable resources and slow the communication processes.

Upon receiving the notification from the downstream nodes, the corresponding upstream node will check its routing table and delete the corresponding entry of the affected node. This upstream node will in turn forward the notification message to its 1-hop neighbour. The process is repeated until the notification reaches the source node. The source node will perform similar tasks and delete the entry of that particular node from its table so that it will not send further incoming packets through the corrupted node.

Traffic diversion. Each intermediate node knows the position and the shortest distance to their 1-hop neighbour. This information is obtained through periodic beacon updates between nodes. At this point, a node's routing table will only contain a fresh list of its 1-hop unaffected neighbours. This will be the nodes located outside the boundaries of the affected regions and thus will be able to forward the packets to the correct destinations. In order to send a packet, a 1-hop neighbour with the closest geographical distance to destination will be chosen. The intermediate nodes, upon receiving the packets, will in turn find their 1-hop neighbour and continue forwarding the packets. Unless they receive another infection notification, these processes repeated until all the packets reach their final destinations.

The identification of infected nodes prior to transmission is crucial to discover the way to divert the incoming traffic away from the infected areas. Since all the selected nodes are free from visiting the infected region, this method avoids the packets from being trapped in there and lost. This saves considerable time and resources for retransmissions and ensures that packets are sent with the least possible delay. There will be minimum communication overheads since it only requires the knowledge of the node's 1-hop neighbour. If, in any case that this approach meets the local minima problem, the first approach explained in Section 4.2.1 is automatically applied.

Beacon updates. Current updates on delivery status are very important in observing a timely data delivery in WSN. As such, we limit the updates to every five intermediate nodes in order to reduce routing overheads. We tested and justified the impact of choosing a particular amount of

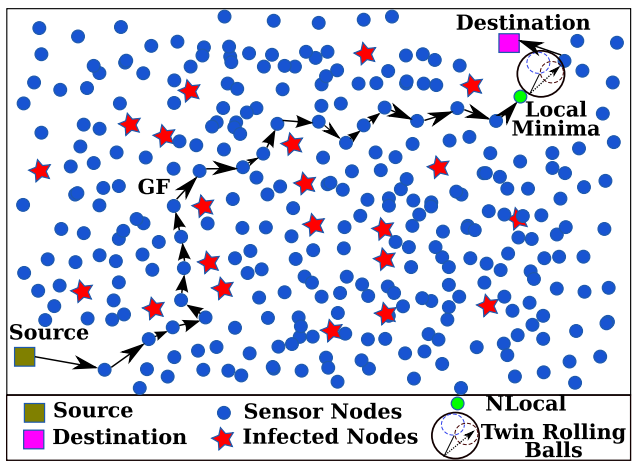

Fig. 9. Infection area and traffic diversion scenario.

updates in the evaluation section. If the ACK is not received from the fifth node after a certain threshold, the source node will retransmit the same packet from which the ACK is missing.

The source node will piggyback any received infection notification message to downstream nodes. The first node that receives the notification will change its next hop information and send the packets to the next available hop in its routing table. Nevertheless, considering a larger duration in sending the ACK (e.g. 10th nodes) may lead to considerable delay in detecting errors, thus waste substantial energy. For example, if the first node is infected during transmission, it can only be detected after the 10th node. Therefore, there will be huge waste of resources in terms of energy and transmission time that could be otherwise spent on more accurate and valuable information. This may also result in obsolete information which is critical in WSN and may cost human lives.

\subsubsection{Normal Forwarding Algorithm}

In a normal condition when there is no infected node, all the packets are forwarded using the normal GF algorithm which is based on the closest 1-hop neighbour. The source node, knowing the address of the destination node, will encapsulate the information of destination node in the packets to its 1-hop neighbour. Based on the information specified in the received packet, the current node learns the address of the destination node and forwards the packets to its next 1-hop neighbour. These processes repeat until the packets reach their destination. In the case of local minima, the BPR technique will be automatically applied.

Fig. 9 shows a scenario of infected nodes and how the proposed method diverts the packets away from the identified regions. Note that once infected, the node will send flag notification to source node so that the corresponding infected node will be deleted from its neighbouring tables. The proposed RB will be applied when local minima is met.

\section{Proof of Correctness}

This section provides the proof for the proposed solution in mitigating Problem 1 for local minima and Problem 2 for false boundary detection in the existing rolling ball mechanism.

Theorem 1 (Avoiding Local Minima Problem). During local minima problem where $d\left(P_{N_{v}}, P_{N_{D}}\right)<d\left(P_{N_{i}}, P_{N_{D}}\right)$ for all $N_{i} \in i$ with the angle covered by $\angle N_{i} N_{v} N_{X}$, then $N_{v}$ is the 
local minima node $\left(N_{\text {Local }}\right)$ and $N_{X}$ is assigned as a potential exit gate node within the coverage of $N_{\text {Local }}$. This is based on the following argument.

Proof. The BPR protocol is automatically applied at the point where local minima problem occurs $\left(N_{\text {Local }}\right)$. The proposed BPR suggests that the rolling ball moves in both directions so we can find the shortest distance of the next hop to destination. To justify, assume that the ball just moves in one direction $d_{c}$ and it happens that the node found in the other direction $d_{c c}$ is closer than the one in $d_{c}$, then the first node $\left(N_{i}\right)$ that hits the ball will not be the closest next hop $\left(d\left(P_{N_{v}}, P_{N_{D}}\right)<d\left(P_{N_{i}}, P_{N_{D}}\right)\right)$ to destination. Therefore, proposing two rolling balls which rotate in opposite directions is a better approach. This is shown in Fig. 8 from which we have proven that (see Definition 1) the proposed BPR method is able to overcome the local minimal problem while finding the next available shortest route. Performance evaluation also discovered that this method detects the next boundary node faster than the existing rolling ball mechanism.

Theorem 2 (By-Passing Infected region). In order to by-pass the infected region, an Exit Gate Node $\left(N_{\text {exit }}\right)$ should be assigned. Here, the $N_{\text {exit }}$ should be located within the coverage of the local minima node $N_{\text {Local }}$ so that it will avoid traversing unnecessary paths which are located too far from destination node.

Proof. The algorithm proposed in BPR technique helps prevent unnecessary use of longer routes and thus saves considerable resources. For this reason, the $N_{\text {exit }}$ is selected within the $N_{\text {Local }}$ communication range $R$ where the rolling ball intersects or hits the last node before the looping occurs as shown in Fig. 8. If in any case where the $N_{\text {exit }}$ is outside this range, a longer route will result (e.g. $N_{e}$ as in rolling ball technique). In our method, the $N_{\text {exit }}$ will perform the normal GF algorithm in choosing the next forwarding hop in such a way that $d\left(N_{\text {exit }}, N_{d}\right)<d\left(N_{k}, N_{d}\right) \forall N_{k} \in V\left(N_{\text {Local }}\right)$ where the other neighbouring nodes are further away than itself. In this case, the new hop should satisfy $d\left(N_{\text {new Hop }}, N_{d}\right)<d\left(N_{\text {exit }}, N_{d}\right)$ with a closer distance to the destination node compared with the $N_{\text {exit }}$.

\section{EXPERIMENTAL Evaluation}

In this section, we evaluate the performance of BPR through NS-2 simulations using some pre-defined metrics. To rate the performance, we compare the performance of our result with BOUNDHOLE and GAR approaches using the configuration setup shown in Table 2. Our simulation is based on a configuration where 100 to 500 nodes, are randomly scattered in a monitored region of $1,000 \mathrm{~m} \times 1,000 \mathrm{~m}$. The sensor nodes perform continuous information sensing while sending periodic updates to the sink node.

\subsection{Number of Hops}

Performance comparisons between our approach, BOUNDHOLE and GAR are shown in Fig. 10a. As the area of infected nodes increases, the number of hops that the packets have to travel also increase considerably, both in sparse and dense networks. GAR gives better performance than
TABLE 2

Simulation Setup

\begin{tabular}{lll}
\hline No & Input Parameters & Setup \\
\hline 1 & Area of Sensor Field & $1,000 \times 1,000 \mathrm{~m}$ \\
2 & Number of Sensor Nodes & $100-500$ \\
3 & Number of Sink Node & 1 \\
4 & Bandwidth & $250 \mathrm{kbps}$ \\
5 & Packet Size & $30 \mathrm{bytes}$ \\
6 & Simulation time & $1,000 \mathrm{~s}$ \\
7 & Radio Propagation Model & TwoRayGround \\
8 & Antenna & OmniAntenna \\
9 & Frequency Band & $2.4 \mathrm{GHz}$ \\
10 & Transmission Range & 50 meters \\
11 & Energy Model & Battery \\
\hline
\end{tabular}

BOUNDHOLE due to the used of the rolling ball approach that help limits the number of traversed hops.

However, our approach exhibits a much better performance than GAR with a further reduction in the number of hops traversed even in bigger numbers of infected nodes. Although the overall trend is increasing, the number of hops visited using the proposed approach is still much more smaller than BOUNDHOLE and GAR. This is a good performance indicator as it reflects that our method can find the shortest route to destination.

Fig. 10b shows the same studies for a dense network. Interestingly, the performance of the BOUNDHOLE and GAR are similar to BPR at the very beginning. As the area grows, starting from 300 meter, the resulting hop counts for BOUNDHOLE drastically increases, reaching up to 30 nodes. This has marked a good differentiation with our solution which found a 'short-cut' route and diverted the packets through uninfected nodes that are closer to those routed using the BOUNDHOLE method.

\subsection{Packet Delivery Ratio}

Fig. 10c shows the ratio of the packets that are successfully delivered to destinations. In BOUNDHOLE, the looping condition prevented the packets from being sent outside the region, so could not be received by the destination and thereby lowering the percentage of successful packet deliveries. That explains the drop in delivery ratio in a larger area.

In contrast, our BPR and GAR methods exhibit much better performance than BOUNDHOLE. This is because of the proper arrangement that has been made to find the exit gate node $\left(N_{\text {exit }}\right)$ that can forward the packets to the outside, unaffected nodes. However, wrong selections of the $N_{\text {exit }}$ limits GAR performance in achieving high performance in comparison to our technique. We strongly believe that our proposed route diversion method greatly helps in reducing the amount of stuck packets, thus lower the loss rate and increase the delivery ratio.

Therefore, an increase in the percentage of infected nodes as evident from the increase in infected area, results in a slight drop of PDR, but this decline is still within an acceptable range of real-time threshold. The higher rate of delivery ratio is as expected for the smaller rate of infection since the amount of packet loss is limited and all other uninfected nodes can easily transmit their packets to the destination node. 


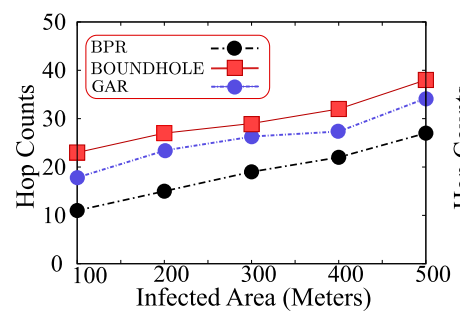

(a)

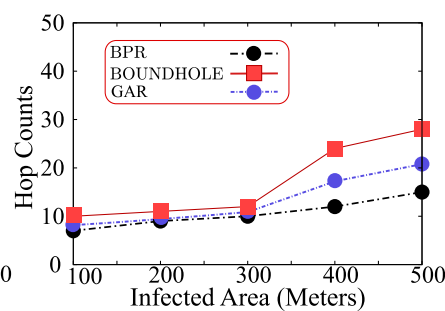

(b)

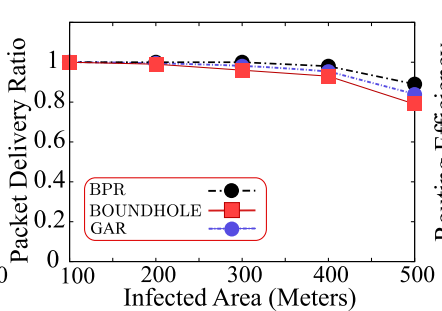

(c)

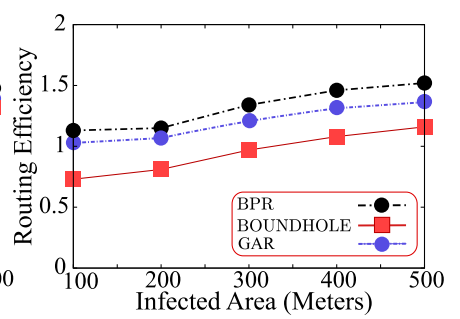

(d)

Fig. 10. The resulting (a) Number of hops in sparse network. (b) Number of hops in dense network. (c) Packet delivery ratio. (d) Routing efficiency.

\subsection{Routing Path Efficiency}

This metric is defined as the ratio of the number of hops for the entire networks to the number of hop counts using the shortest distance. The resulting routing efficiency is illustrated in Fig. 10d. The path efficiency for the BOUNDHOLE scheme increases only gradually, owing to the occurrence of the routing loop, resulting in more hops used. Although our method exhibits a similar trend in larger infected areas, the resulting path efficiency is much higher. This proves that the proposed technique is able to provide better performance than existing BOUNDHOLE and GAR methods. In particular, this shows our ability to find a shorter path and avoid falling into the looping problem or visit unnecessary nodes. This has increased our path efficiency compared to BOUNDHOLE and GAR techniques.

\subsection{Energy Consumption}

Figs. 11a and $11 \mathrm{~b}$ plots the percentage energy consumption obtained through simulation using the same configuration in Table 2. These are the energy used for data transmission and any dropped bits. As with the other two metrics, we investigated the energy spent in sparse and dense networks and compared the performance with BOUNDHOLE and GAR. The figure clearly shows that the energy consumption in a sparse network is much higher than in the dense network. This is due to fewer hops that can be used to transfer packets, requiring each node to utilize more energy to transfer packets to destination. The increase in the infected area has also increased the average energy spent for both protocols. Similar patterns can be seen in the dense network, but with much lower energy for both methods.

Nevertheless, the average energy spent using our method is far below the BOUNDHOLE and GAR methods in both scenarios. There is a sudden increase in energy in BOUNDHOLE when the infected area is larger than 300 meters. This drastic change is due to the sudden increase of the trapped packets which require further retransmission processes that can incur substantial expenses of energy. This is also mainly driven by improper data dissemination processes while handling the infected data, causing high energy consumption which quickly drains nodes' energy and shortens network lifetime. Efficiently getting the stuck packets out of the infected regions, we have reduced the average energy consumption for retransmission of the lost packets.

\subsection{End-To-End Delay}

The resulting end-to-end delay can be seen in Figs. 11c and $11 \mathrm{~d}$. In both sparse and dense scenarios, the proposed BPR technique shows remarkable performance with over 51.5 and 72.2 percent improvements respectively compared with BOUNDHOLE and GAR. This is closely related to the resulting number of hops and the packet delivery ratio. The longer routes in BOUNDHOLE and GAR have resulted in higher end-to-end delay as the packets need to traverse longer paths and thus increase the average waiting time. As we have found a better way to route the packets out from the infected regions, the average end-to-end delay for our method is much lower than both alternative methods in either scenario.

\subsection{Throughput}

Fig. 12a presents the throughput gained with the change in offered load. The results are shown with a 99 percent confidence interval. As shown in the figure, there is huge throughput difference between BPR and BOUNDHOLE from 50,000 offered load upwards. This substantial gap reflects a high percentage of packets trapped or lost, thus affecting the associated throughput in BOUNDHOLE. There is also a significant drop of throughput in the BOUNDHOLE method at the final point, making a huge gap between both studied methods. Having said that, the proposed BPR method always demonstrates better performance regardless of any condition.

\subsection{Communication Overheads}

Fig. 12b shows performance comparison of communication overhead (routing packets) versus sensor node density for all techniques. As the number of nodes increase with greater network density (the infected area), massive routing packets are required to route packets to their destination nodes. High communication overheads may affect performance as they add up the number of network traffic, exposing it to a high possibility of congestion and network collision.

The low number of visiting hops in the BPR method involves lower communication overheads. This contrasts starkly with the sweeping line approach used in BOUNDHOLE and the rolling ball method in GAR. Referring to GAR's performance, this can be attributed to the large number of visited hops, resulting in much greater communication overheads. In BOUNDHOLE, its looping problem developed a huge amount of routing overheads since it involves more hops for the packets to reach their destinations. An increased trend is seen in the larger infected area for all methods. However, the increase in the infected area does not have so much impact on our BPR method as compared to BOUNDHOLE which shows a sudden increase from 300 meters onwards. 


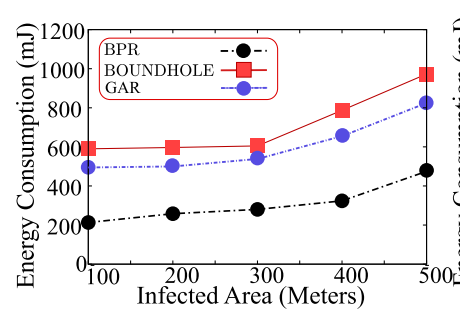

(a)

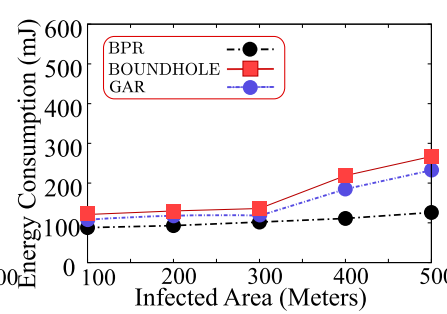

(b)

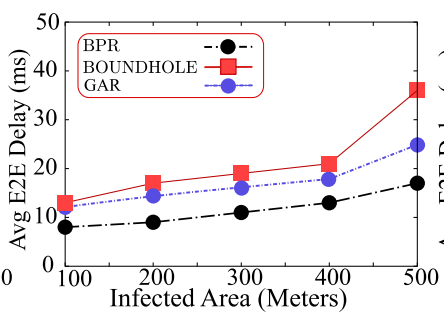

(c)

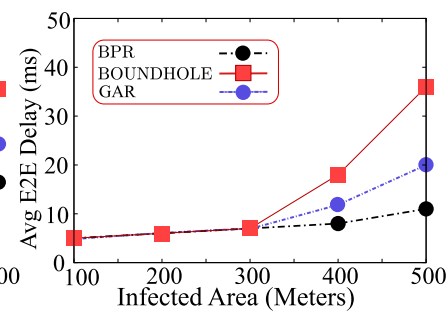

(d)

Fig. 11. The resulting (a) Energy consumption in sparse network. (b) Energy consumption in dense network. (c) Average end-to-end delay in sparse network. (d) Average end-to-end delay in dense network.

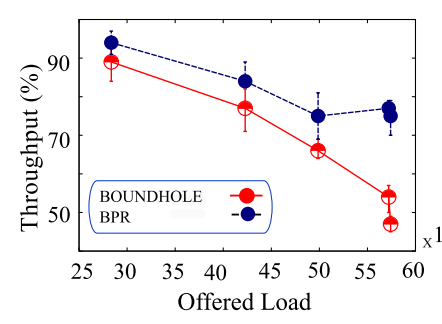

(a)

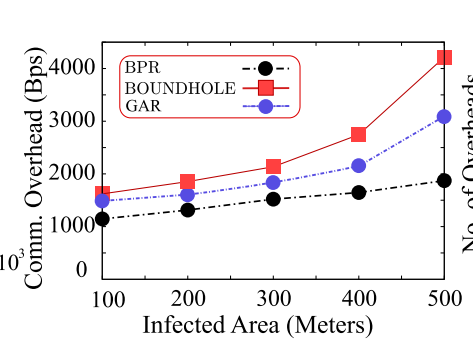

(b)

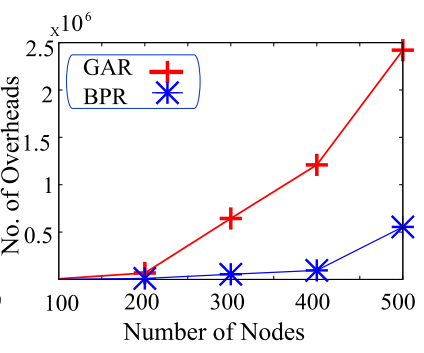

(c)

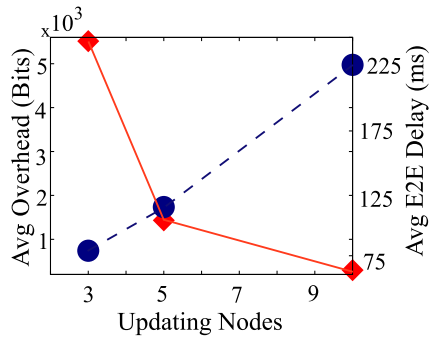

(d)

Fig. 12. The resulting (a) Throughput. (b) Communication overhead. (c) Number of overheads versus number nodes. (d) Performance trade-off between average overhead, number of updating nodes and end-to-end delay.

\subsection{Overhead versus Number of Nodes}

Here we testify to the effectiveness of our method using the resulting overheads, measured against the number of nodes. The performance is compared with the GAR technique. Due to the efficient routing approach, the obtained overheads in BPR are far below the GAR technique. This can be seen in Fig. 12c. In addition, the method of sending the ACK from every fifth intermediate node to the source node further helps to reduce total overheads. This method allows traffic reduction and reduces the amount of ACK while at the same time keeping the status up-to-date. This can also be proved by the number of average overheads in Fig. 12d.

\subsection{Performance Trade-Off: Average Overhead versus Number of Intermediate Updating Nodes}

In regular WSN operations, sensor nodes will send updates or acknowledgements back to the source node for every packet received. Although regular updates (e.g. every three nodes) may keep the system updated with the latest network state, it causes a significant amount of overhead which quickly depletes nodes' energy. Furthermore, this scenario accumulates more traffic and reduces the entire network performance.

To cater to this issue, we introduce a new technique that sends the ACK to the source nodes every fifth intermediate node. The reason for choosing this number is shown in Fig. 12d plotted against the resulting average end-to-end delay. If we increase the number of updating nodes, there will be a huge performance trade-off that increases the average end-to-end delay. Due to the performance imbalance, we take into account a figure that can constitute and balance both measurements for optimum performance. Therefore, updates are suggested for every five intermediate nodes.

Finally, we compare the overall performance of our approach with GAR and BOUNDHOLE as shown in Table 3.
TABLE 3

Summarized Performance Comparison

\begin{tabular}{llll}
\hline Metrics/Protocols & BPR & GAR & BDHOLE \\
\hline False Boundary Detection & NO & NO & YES \\
Unnecessary Visits & NO & YES & YES \\
Routing Efficiency & HIGH & MED & LOW \\
Energy Consumption & LOW & MED & HIGH \\
Communication Overhead & LOW & MED & HIGH \\
Previous hole's knowledge & NO & NO & YES \\
Memory requirement & LOW & MED & HIGH \\
Avoid Local Minima & YES & YES & YES \\
Shortest Path & SHORTEST & MED & LONGEST \\
Congestion-free & YES & NO & NO \\
Algorithm Complexity & LOW & MED & HIGH \\
\hline
\end{tabular}

This study validates the significant contribution of BPR which considers factors that are absent in the other two protocols: the twin rolling balls and the elimination of unnecessary visits to other nodes. These two achievements help us nail the high performance in various metrics, resulting in exceptional performance.

\section{CONCLUSION}

In this paper, we have studied the effectiveness of our proposed by-passed routing technique in avoiding infected areas and its efficacy in improving the overall performance. The infected areas are anomalous nodes detected using a fuzzy data clustering method and the information collected is used in the proposed BPR technique. With this mechanism, we have solved three major dilemmas in the traditional routing approaches: local minima, false boundary detection and visits to unnecessary nodes.

We evaluated BPR using different scenarios in NS-2 and have proven it to exhibit high performance compared with 
the other studied protocols, BOUNDHOLE and GAR. The proposed twin rolling balls greatly help to define the next forwarding node and mitigate the false boundary detection applicable in the existing rolling ball technique. The introduction of dynamic routing greatly helps to minimize the possibility of false route diversion that may lead to substantial packet loss and long delays. We also have a different method of selecting the exit gate node which shortens the forwarding path to the destination node. Overall evaluation shows favourable and promising performance improvement over previous methods.

\section{REFERENCES}

[1] A. Abdulla, H. Nishiyama, J. Yang, N. Ansari, and N. Kato, "HYMN: A novel hybrid multi-hop routing algorithm to improve the longevity of WSNs," IEEE Trans. Wireless Commun., vol. 11, no. 7, pp. 2531-2541, Jul. 2012.

[2] M. Ahmadi Livani and M. Abadi, "An energy-efficient anomaly detection approach for wireless sensor networks," in Proc. 5th Int. Symp. Telecommun., 2010, pp. 243-248.

[3] N. Ahmed, S. S. Kanhere, and S. Jha, "The holes problem in wireless sensor networks: A survey," SIGMOBILE Mob. Comput. Commun. Rev., vol. 9, no. 2, pp. 4-18, Apr. 2005.

[4] I. Akyildiz, W. Su, Y. Sankarasubramaniam, and E. Cayirci, "A survey on sensor networks," IEEE Commun. Mag., vol. 40, no. 8, pp. 102-114, Aug. 2002.

[5] N. Arad and Y. Shavitt, "Minimizing recovery state in geographic ad hoc routing," IEEE Trans. Mobile Comput., vol. 8, no. 2, pp. 203217, Feb. 2009

[6] Ashwini and A. S., "Information dissemination between nodes of different intersections intersection in city environment using hop greedy routing protocol (BAHG)," Int. J. Ethics Eng. Manag. Educ., vol. 1, no. 4, pp. 232-236, Apr. 2014.

[7] D. Chen and P. K. Varshney, "On-demand geographic forwarding for data delivery in wireless sensor networks," Comput. Commun., vol. 30, no. 1415, pp. 2954-2967, 2007.

[8] S. Chen, G. Fan, and J. hong Cui, "Avoid 'void' in geographic routing for data aggregation in sensor networks," Int. J. Ad Hoc Ubiquitous Comput., vol. 1, pp. 169-178, 2006.

[9] R. Di Pietro, L. Mancini, C. Soriente, A. Spognardi, and G. Tsudik, "Data security in unattended wireless sensor networks," IEEE Trans. Comput., vol. 58, no. 11, pp. 1500-1511, Nov. 2009.

[10] Q. Fang, J. Gao, and L. Guibas, "Locating and bypassing holes in sensor networks," Mobile Netw. Appl., vol. 11, no. 2, pp. 187-200, 2006.

[11] K.-I. Kim, M.-J. Baek, and T.-E. Sung, "Load balancing for greedy forwarding of geographic routing in wireless networks," IEICE Trans., vol. 93-B, no. 8, pp. 2184-2187, 2010.

[12] H. Kumarage, I. Khalil, Z. Tari, and A. Zomaya, "Distributed anomaly detection for industrial wireless sensor networks based on fuzzy data modelling," J. Parallel Distrib. Comput., vol. 73, no. 6, pp. 790-806, Jun. 2013.

[13] S. Lai and B. Ravindran, "Least-latency routing over time-dependent wireless sensor networks," IEEE Trans. Comput., vol. 62, no. 5, pp. 969-983, May 2013.

[14] W. Liu, H. Nishiyama, N. Ansari, J. Yang, and N. Kato, "Clusterbased certificate revocation with vindication capability for mobile ad hoc networks," IEEE Trans. Parallel Distrib. Syst., vol. 24, no. 2, pp. 239-249, Feb. 2013.

[15] J. Na, D. Soroker, and C.-K. Kim, "Greedy geographic routing using dynamic potential field for wireless ad hoc networks," IEEE Commun. Lett., vol. 11, no. 3, pp. 243-245, Mar. 2007.

[16] H. Nakayama, Z. Fadlullah, N. Ansari, and N. Kato, "A novel scheme for WSAN sink mobility based on clustering and set packing techniques," IEEE Trans. Autom. Control, vol. 56, no. 10, pp. 2381-2389, Oct. 2011.

[17] H. Nakayama, N. Ansari, A. Jamalipour, and N. Kato, "Faultresilient sensing in wireless sensor networks," Comput. Commun., vol. 30, no. 11-12, pp. 2375-2384, Sep. 2007.

[18] H.-T. Pai and Y. S. Han, "Power-efficient direct-voting assurance for data fusion in wireless sensor networks," IEEE Trans. Comput., vol. 57, no. 2, pp. 261-273, Feb. 2008.
[19] P. Ponnavaikko, S. Wilson, and J. Holliday, “Greedy least interference forwarding algorithm using directional antennas for cellular ad-hoc networks," in Proc. Military Commun. Conf., 2010, pp. 1484-1489.

[20] L. Popa, C. Raiciu, I. Stoica, and D. Rosenblum, "Reducing congestion effects in wireless networks by multipath routing," in Proc. IEEE Int. Conf. Netw. Protocols, 2006, pp. 96-105.

[21] G. Q. Fang, J. Gao, and L. J.,“'Locating and bypassing routing holes in sensor networks," in Proc. 23rd Annu. Joint Conf. IEEE Comput. Commun. Soc., 2004, pp. 2458-2468.

[22] P. Sakarindr and N. Ansari, "Security services in group communications over wireless infrastructure, mobile ad hoc, and wireless sensor networks," IEEE Wireless Commun., vol. 14, no. 5, pp. 8-20, Oct. 2007.

[23] S. Subramanian, S. Shakkottai, and P. Gupta, "On optimal geographic routing in wireless networks with holes and non-uniform traffic," in Proc. IEEE 26th Int. Conf. Comput. Commun., May 2007, pp. 1019-1027.

[24] S. Tang and B. Mark, "Analysis of virus spread in wireless sensor networks: An epidemic model," in Proc. 7th Int. Workshop Des. Reliable Commun. Netw., 2009, pp. 86-91.

[25] K.-T. F. Wen-Jiunn Liu, "Greedy routing with anti-void traversal for wireless sensor networks," IEEE Trans. Mobile Comput., vol. 8, no. 7, pp. 910-922, Jul. 2009.

[26] W. Zhang, Q. Yin, H. Chen, F. Gao, and N. Ansari, "Distributed angle estimation for localization in wireless sensor networks," IEEE Trans. Wireless Commun., vol. 12, no. 2, pp. 527-537, Feb. 2013.

[27] Y. Zhang, S. He, J. Chen, Y. Sun, and X. Shen, "Distributed sampling rate control for rechargeable sensor nodes with limited battery capacity," IEEE Trans. Wireless Commun., vol. 12, no. 6, pp. 3096-3106, Jun. 2013.

[28] L. Zou, M. Lu, and Z. Xiong, "A distributed algorithm for the dead end problem of location based routing in sensor networks," IEEE Trans. Veh. Technol., vol. 54, no. 4, pp. 1509-1522, Jul. 2005.

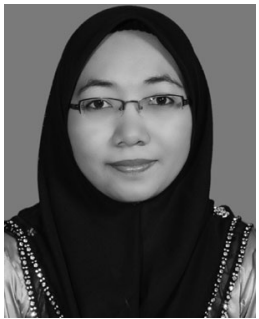

Naimah Yaakob received the MSc degree in computer and information engineering from the International Islamic University, Malaysia (IIUM) in 2008. She submitted the PhD thesis at the School of Computer Science and Information Technology, RMIT University, Australia. She is currently with the School of Computer and Communication Engineering, Universiti Malaysia Perlis. Her research interests include wireless sensor networks, congestion control in distributed networking systems and pervasive communication in healthcare environment.

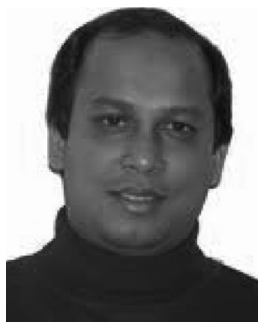

Ibrahim Khalil received the PhD degree in 2003 from University of Berne, Switzerland. $\mathrm{He}$ is a senior lecturer in the School of Computer Science and Information Technology, RMIT University. He has several years of experience in Silicon Valleybased companies working on Large Network Provisioning and Management software. Before joining RMIT, he was with EPFL and the University of Berne in Switzerland and Osaka University in Japan. His research interests are anonymous networks, quality of service, wireless sensor networks, and remote healthcare.

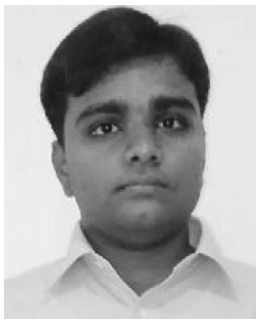

Heshan Kumarage received the BSc (hons) degree in computer science and engineering from the University of Moratuwa, Sri Lanka. in 2009. He was an assistant lecturer at the Sri Lanka Institute of Information Technology from 2009 to 2010 . He is currently working toward the PhD degree at RMIT University, Melbourne, Australia. His research interests include distributed systems, data mining for network security, information theory, and wireless sensor networks. 


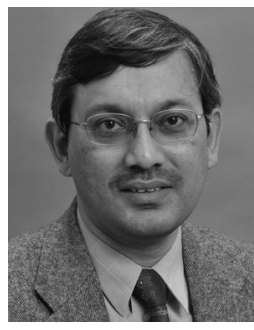

Mohammed Atiquzzaman received the MS and $\mathrm{PhD}$ degrees in electrical engineering and electronics from the University of Manchester, United Kingdom. He currently holds the Edith Kinney Gaylord Presidential professorship in the School of Computer Science at the University of Oklahoma. He is the editor-in-chief of Journal of Networks and Computer Applications, founding editor-in-chief of Vehicular Communications and has served/serving on the editorial boards of various IEEE journals and co-chaired numerous IEEE international conferences including IEEE Globecom. His research interests are in communications switching, transport protocols, wireless and mobile networks, satellite networks, and optical communications. $\mathrm{He}$ is a senior member of the IEEE.

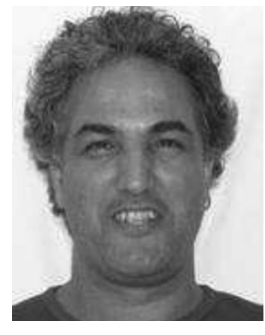

Zahir Tari received the honors degree in operational research from the Universite des Sciences et de la Technologie Houari Boumediene (USTHB), Algiers, Algeria, the master's degree in operational research from the University of Grenoble I, France, and the $\mathrm{PhD}$ degree in artificial intelligence from the University of Grenoble II, France. He is the head at the Distributed Systems and Networking Discipline, School of Computer Science and Information Technology, RMIT University. His current research focuses on web servers and SOAP-based systems, SCADA system security, and web services. He has organized more than 12 international conferences and is a senior member of the IEEE.

$\triangle$ For more information on this or any other computing topic, please visit our Digital Library at www.computer.org/publications/dlib. 